PROCEEDINGS OF THE

AMERICAN MATHEMATICAL SOCIETY

Volume 130, Number 5, Pages 1249-1256

S 0002-9939(01)06441-3

Article electronically published on December 20, 2001

\title{
A GENERALIZATION OF THE NON-TRIVIALITY THEOREM OF SERRE
}

\author{
STEPHAN KLAUS
}

(Communicated by Ralph Cohen)

\begin{abstract}
We generalize the classical theorem of Serre on the non-triviality of infinitely many homotopy groups of 1-connected finite CW-complexes to CW-complexes where the cohomology groups either grow too fast or do not grow faster than a certain rate given by connectivity. For example, this result can be applied to iterated suspensions of finite Postnikov systems and certain spaces with finitely generated cohomology ring. In particular, we obtain an independent, short proof of a theorem of R. Levi on the non-triviality of $k$ invariants associated to finite perfect groups. Another application concerns spaces where the cohomology grows like a polynomial algebra on generators in dimension $n, 2 n, 3 n, \ldots$ for a fixed number $n$. We also consider spectra where we prove a non-triviality result in the case of fast growing cohomology groups.
\end{abstract}

A classical theorem of J.P. Serre (for $p=2,[\mathrm{~S}]$ ) and Y. Umeda (for odd $p,[\mathrm{U}]$ ) states that a 1-connected finite $\mathrm{CW}$-complex $X$ with non-trivial cohomology mod $p$ has infinitely many non-trivial homotopy groups mod $p$.

In [MN], C.A. McGibbon and J.A. Neisendorfer proved a stronger result (the existence of $p$-torsion elements in infinitely many dimensions for finite dimensional spaces) using H. Miller's theorem on the Sullivan conjecture.

Using the same methods from analytical number theory as in the original proof of Serre, we show a straightforward generalization of the Serre non-triviality theorem to certain infinite CW-complexes, which seems not to be in the literature. The point is that the cohomology of a finite Postnikov section has a certain growth rate depending of the highest homotopy group. Thus, spaces where cohomology grows faster or slower cannot have a finite Postnikov system. We need some notation:

Definition 1. Let $X$ be a connected CW-complex and $p$ a prime. We call $X$ of finite type if $H_{k}(X ; \mathbb{Z})$ is finitely generated for all $k>0$. Then we can define the mod $p$ Poincaré series as the formal power series with integer coefficients

$$
\begin{gathered}
h(X, t):=\sum_{k} h^{k}(X) t^{k}, \\
h^{k}(X):=\operatorname{dim} H^{k}(X ; \mathbb{Z} / p) .
\end{gathered}
$$

Received by the editors January 6, 2000 and, in revised form, July 26, 2000.

1991 Mathematics Subject Classification. Primary 19D06, 20J05, 20J06, 55P20, 55P40, 55P42, 55P60, 55Q52, 55R35, 55S45, 55T10.

Key words and phrases. Homotopy group, Postnikov system, $k$-invariant, Poincaré series, growth rate, suspension, Hilbert-Serre theorem, group cohomology, perfect group, $p$-completion, Quillen plus construction, stable homotopy group. 
We denote by

$$
\rho(X) \in \mathbb{R}^{+} \cup \infty
$$

its radius of convergence, which is given by $\rho(X)=\left(\limsup _{k} \sqrt[k]{h^{k}(X)}\right)^{-1}$. The connectivity $\bmod p$ of $X$, denoted by

$$
\operatorname{conn}(X) \in \mathbb{N} \cup \infty,
$$

is defined as the largest integer $n$ such that $h^{k}(X)=0$ for all $1 \leq k \leq n$. If $\rho(X) \geq 1$, following Serre we define a positive real function $\phi(X,-): \mathbb{R}^{+} \rightarrow \mathbb{R}^{+}$ measuring the growth of $h(X, t)$ for $t \rightarrow 1^{-}$by

$$
\phi(X, s):=\log _{p} h\left(X, 1-p^{-s}\right) .
$$

We define the logarithmic growth rate $\bmod p$ of $X$,

$$
\lg (X) \in \mathbb{N} \cup \infty,
$$

as the largest integer $r$ such that

$$
\lim _{s \rightarrow \infty} \phi(X, s) / s^{r} \neq 0 .
$$

These invariants have the following obvious properties: If $\rho(X)>1$, then $\rho(X)=$ $\infty$ because then $H^{*}(X ; \mathbb{Z} / p)$ has to be finite dimensional. In this case, $\lg r(X)=0$. If $h^{k}(X) \leq h^{k}(Y)$ for all $k$, then $\rho(X) \geq \rho(Y)$ and $h(X, t) \leq h(Y, t)$ for all $0 \leq t<$ $\rho(Y)$. If $\rho(Y) \geq 1$, then also $\phi(X, s) \leq \phi(Y, s)$ for all $s \geq 0$.

If $F \rightarrow E \rightarrow B$ is a fibration of 1-connected spaces of finite type, the Serre spectral sequence gives $h^{n}(E) \leq \sum_{p+q=n} h^{p}(F) h^{q}(B)$. Hence $\rho(E) \geq \min (\rho(F), \rho(B))$ and

$$
\begin{gathered}
h(E, t) \leq h(F, t) h(B, t), \\
\phi(E, s) \leq \phi(F, s)+\phi(B, s)
\end{gathered}
$$

on the common domain of definition. If $\rho(F) \geq 1$ and $\rho(B) \geq 1$, then $\operatorname{lgr}(E) \leq$ $\max (\lg r(F), \operatorname{lgr}(B))$.

The following analytical result of Serre (for $p=2$ ) and Umeda (for odd primes p) is basic:

Theorem 1 ([S], Theorem 10; [U]). The mod p Poincaré series of EilenbergMacLane spaces has a radius of convergence equal to 1 with growth rates given by

$$
\begin{gathered}
\lim _{s \rightarrow \infty} \phi\left(K\left(\mathbb{Z} / p^{m}, n\right), s\right) / s^{n}=1 / n !, \\
\lim _{s \rightarrow \infty} \phi(K(\mathbb{Z}, n), s) / s^{n-1}=1 /(n-1) ! \quad(n \geq 2) .
\end{gathered}
$$

Hence for a non-trivial finitely generated group $\pi$,

$$
\operatorname{lgr}(K(\pi, n))= \begin{cases}n-1 & \text { if } \pi \text { is free, } \\ n & \text { if } \pi \text { is not free. }\end{cases}
$$

Now we prove our main result:

Theorem 2. Let $X$ be a 1-connected $C W$-complex of finite type.

1. If $\rho(X)<1$, or $\rho(X)=1$ and $\lg (X)=\infty$, then $\pi_{*}(X) \otimes \mathbb{Z} / p$ is non-trivial in arbitrarily high dimensions. 
2. If $\rho(X) \geq 1$ and $\operatorname{lgr}(X)<\operatorname{conn}(X)$ this also holds true. Here, even the stronger conclusion holds that the Postnikov tower for $X$ has infinitely many non-vanishing $k$-invariants. In the case that all $H^{k}(X ; \mathbb{Z})$ are finite $(k \geq 2)$, this is also true for $\lg r(X)=\operatorname{conn}(X)$.

Proof. Let $Y$ be a 1-connected Postnikov tower with finitely generated homotopy groups $\pi_{k}$ satisfying that $\pi_{k} \otimes \mathbb{Z} / p=0$ for $1 \leq k \leq r$ and for $k>N$ (for some $r, N$ with $r<N)$, but $\pi_{k} \otimes \mathbb{Z} / p$ is non-trivial for $k=r+1$ and for $k=N$. In particular, $\operatorname{conn}(Y)=r$. On the one hand, the Postnikov tower $K\left(\pi_{k+1}, k+1\right) \rightarrow Y^{(k+1)} \rightarrow$ $Y^{(k)}$ together with $Y^{(r+1)}=K\left(\pi_{r+1}, r+1\right)$ gives

$$
h(Y, t) \leq h\left(K\left(\pi_{r+1}, r+1\right), t\right) \cdots h\left(K\left(\pi_{N}, N\right), t\right) .
$$

Thus $\rho(Y) \geq 1$ and

$$
\phi(Y, s) \leq \phi\left(K\left(\pi_{r+1}, r+1\right), s\right)+\ldots+\phi\left(K\left(\pi_{N}, N\right), s\right) .
$$

On the other hand, the Whitehead tower $K\left(\pi_{k+1}, k\right) \rightarrow Y\langle k+1\rangle \rightarrow Y\langle k\rangle$ of $k$ connected coverings of $Y$ together with $h(Y\langle N-1\rangle, t)=h\left(K\left(\pi_{N}, N\right), t\right)$ implies that

$$
h\left(K\left(\pi_{N}, N\right), t\right) \leq h(Y, t) h\left(K\left(\pi_{r+1}, r\right), t\right) \cdots h\left(K\left(\pi_{N-1}, N-2\right), t\right) .
$$

Thus $\rho(Y)=1$ and

$$
\phi(Y, s) \geq \phi\left(K\left(\pi_{N}, N\right), s\right)-\left(\phi\left(K\left(\pi_{r+1}, r\right), s\right)+\ldots+\phi\left(K\left(\pi_{N-1}, N-2\right), s\right)\right) .
$$

This gives the growth rate of $Y$ :

$$
\operatorname{lgr}(Y)=\lg \left(K\left(\pi_{N}, N\right)\right)= \begin{cases}N-1 & \text { if } \pi_{N} \text { is free, } \\ N & \text { if } \pi_{N} \text { is not free. }\end{cases}
$$

Now we can finish the proof of our theorem:

1. If $\rho(X)<1$, or $\rho(X)=1$ and $\lg r(X)=\infty$, then $X$ cannot have a Postnikov tower $Y$ as above because $\rho(Y)=1$ and $\lg r(Y)<\infty$. In particular, $X$ has nontrivial homotopy groups mod $p$ in arbitrarily high dimensions.

2. If $\rho(X) \geq 1$ and $\lg r(X)<\operatorname{conn}(X)$, then $X$ cannot have a Postnikov tower $Y$ as above because $\operatorname{conn}(X)=\operatorname{conn}(Y)=r \leq N-1 \leq \operatorname{lgr}(Y)$. In the case that all $H^{k}(X ; \mathbb{Z})$ are finite $(k \geq 2)$, this is also not possible for $\operatorname{lgr}(X)=\operatorname{conn}(X)$ because $\operatorname{conn}(X)=\operatorname{conn}(Y)=r<N=\lg r(Y)$. This shows again that $X$ has non-trivial homotopy groups mod $p$ in arbitrarily high dimensions. Furthermore, assume that only finitely many $k$-invariants of $X$ are non-trivial. Then we find in some dimension $M>>0$ an Eilenberg-MacLane space $K\left(\pi_{M}, M\right)$ with $\pi_{M} \otimes \mathbb{Z} / p$ non-trivial which can be split from $X$ as a direct factor. But, again this contradicts the growth rate of the mod $p$ cohomology of $X$.

We remark that in the first case, all $k$-invariants may be trivial as the example $X:=\prod_{n>r} K(\mathbb{Z} / p, n)$ shows. The infinite wedge of $2^{n}$ copies of the $n$-sphere $S^{n}$ for all $n$ is an example of a space of finite type with $\rho=1 / 2$.

If $\Sigma X$ denotes the suspension of a space, then

$$
\lim _{s \rightarrow \infty} \phi(\Sigma X, s) / s^{r}=\lim _{s \rightarrow \infty} \phi(X, s) / s^{r},
$$

which follows from $h(\Sigma X, t)-1=t(h(X, t)-1)$. Hence, in the case that

$$
\lim _{s \rightarrow \infty} \phi(X, s) / s^{r}=0
$$

for some $r$, we can apply our theorem to a suitable iterated suspension of $X$ : 
Corollary 1. Let $Y$ be a 1-connected Postnikov tower with finitely generated homotopy groups $\pi_{k}$ satisfying that $\pi_{k} \otimes \mathbb{Z} / p=0$ for $1 \leq k \leq r$ and for $k>N$ (for some $r, N$ with $r<N)$, but $\pi_{k} \otimes \mathbb{Z} / p$ is non-trivial for $k=r+1$ and for $k=N$. Then its $n$-fold suspension $\Sigma^{n} Y$, where $n \geq N-r$, has non-trivial homotopy groups $\pi_{*}\left(\Sigma^{n} Y\right) \otimes \mathbb{Z} / p$ in arbitrarily high dimensions. Moreover, the Postnikov tower for $\Sigma^{n} Y$ has infinitely many non-vanishing $k$-invariants. In particular, this is true for any suspension of an Eilenberg-MacLane space $\Sigma^{n} K(\pi, N)$.

Proof. Under the hypotheses, one has $\lim _{s \rightarrow \infty} \phi(Y, s) / s^{N+1}=0$.

Corollary 2. Let $X$ be a 1-connected $C W$-complex such that $H^{k}(X ; \mathbb{Z})$ is finitely generated for all $k \geq 2$, and $H^{*}(X ; \mathbb{Z} / p)$ is finitely generated as an algebra. Additionally assume that $H^{2}(X ; \mathbb{Z} / p)=0$ or that all $H^{k}(X ; \mathbb{Z})$ are finite $(k \geq 2)$. Then $\pi_{*}(X) \otimes \mathbb{Z} / p$ is non-trivial in arbitrarily high dimensions and there are infinitely many non-vanishing $k$-invariants.

Proof. By the Hilbert-Serre theorem ([B], p. 162), the Poincaré series of a finitely generated (graded) commutative algebra $H^{*}$ over $\mathbb{Z} / p$ is given by a rational function of the form

$$
h(t)=\frac{f(t)}{\prod_{j}\left(1-t^{k_{j}}\right)} .
$$

Hence we get

$$
\phi(X, s)=g(s)+l s,
$$

with $g(s):=\log _{p}\left(f(t) / \prod_{j}\left(1+t+t^{2}+\ldots+t^{k_{j}-1}\right), t=1-p^{-s}\right.$, and $l$ is the order of the pole of $h(X, t)$ at $t=1$ (which is equal to the number of factors in the denominator). Clearly, $\lim _{s \rightarrow \infty} g(s)=f(1) / \prod_{j} k_{j}$ is a finite constant. If $H^{2}(X ; \mathbb{Z} / p)=0$, we have $r=2$ and thus $\lim _{s \rightarrow \infty} \phi(X, s) / s^{r}=0$. If all $H^{k}(X ; \mathbb{Z})$ are finite, we have only $r=1$, but $\lim _{s \rightarrow \infty} \phi(X, s) / s^{r+1}=0$. In both cases, our theorem can be applied.

The example of the infinite complex projective space (i.e., $K(\mathbb{Z}, 2)$ ) shows that we need the additional assumption in the corollary above.

The corollary can be applied to classifying spaces $B G$ of 1-connected compact Lie groups $G$, for example to the infinite quaternionic projective space $\left(G=S^{3}\right)$. But this gives nothing new because of the non-triviality theorem for $G$ and $G \simeq \Omega B G$.

More interesting applications are given by suspensions or glueing construction of such spaces, where the growing of cohomology can be controlled by the MayerVietoris sequence. As an example, let $\rho: S U(n) \rightarrow S U(m)$ and $\rho^{\prime}: S U(n) \rightarrow$ $S U\left(m^{\prime}\right)$ be representations, and form the homotopy pushout:

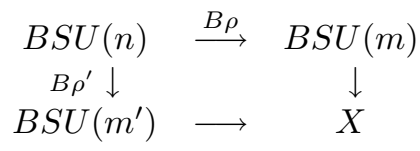

Then the theorem can be applied to $X$.

As another substantial application, we get a variation of a result of $\mathrm{R}$. Levi. In $\mathrm{L}$, it was proved using H. Miller's theorem on the Sullivan conjecture and related results of F. Cohen, J. Lannes and L. Schwartz. 
Recall that a group $G$ is called $p$-perfect for a prime $p$ if $H_{1}(G ; \mathbb{Z} / p)=0$. Then the Bousfield-Kan $p$-completion $B G_{p}^{\wedge}$ of the classifying space of $G$ is a 1-connected $p$ local space with the same mod $p$ cohomology as $B G[\mathrm{BK}]$. For a finite perfect group (i.e., $H_{1}(G ; \mathbb{Z})=0$ ), the $p$-completion is related to the Quillen plus construction by $B G^{+} \simeq \prod_{p} B G_{p}^{\wedge}$.

Corollary 3 ( $\mathrm{L}$, p. 5$)$. Let $G$ be a finite p-perfect group such that $p$ divides the order of $G$. Then the Postnikov tower for $B G_{p}^{\wedge}$ has infinitely many non-vanishing $k$-invariants. In particular, $\pi_{*} B G_{p}^{\wedge}$ is non-trivial in arbitrarily high dimensions.

Proof. We only need the following standard results on the cohomology of a finite group $G$ in order to apply Corollary 2:

Let $G$ be a finite group and $p$ a prime. Then the mod $p$ cohomology ring $H^{*}(B G ; \mathbb{Z} / p)$ is finitely generated as a $\mathbb{Z} / p$-algebra and the integral cohomology groups $H^{k}(B G ; \mathbb{Z}), k>0$, are all finite. The order of the pole of the rational function $h(B G, t)$ at 1 is given by the $p$-rank $r_{p}$ of $G$, i.e., the maximal rank of an elementary abelian $p$-subgroup. (See $[\bar{B}$, Chapters 4 and 5 . Finite generation is a theorem of L. Evens and B.B. Venkov, which gives the rationality of $h(B G, t)$ by the Hilbert-Serre theorem. The order of the pole at 1 is a result of D. Quillen. If $p$ does not divide the order of $G$, then clearly $h(B G, t)=1$.)

We remark that in [L], non-triviality of infinitely many $k$-invariants is proved for $\Omega B G_{p}^{\wedge}$, which is a stronger result. In contrast, our method also applies to the suspensions $\Sigma^{n} B G$ and $\Sigma^{n} B G_{p}^{\wedge}$.

Now we consider spaces with cohomology not finitely generated as an algebra. If $H^{*}$ is a polynomial algebra over $\mathbb{Z} / p$ with the number of algebra generators in degree $n \geq 1$ given by $a_{n} \geq 0$, then the Poincaré series of $H^{*}$ is given by

$$
h(t)=\prod_{n \geq 1}\left(1-t^{n}\right)^{-a_{n}} .
$$

Here, we find it more convenient to consider $\ln h\left(1-e^{-s}\right)$ which is related to $\log _{p} h\left(1-p^{-s}\right)$ by a linear scale transformation.

Lemma 1. Let $f(t), g(t)$ be the formal power series defined by $f(t):=\sum_{n \geq 1} a_{n} t^{n}$ and $g(t):=\sum_{n \geq 1} b_{n} t^{n}$, where $b_{n}:=\sum_{k \mid n} a_{k}$. Then

$$
\ln h(t)=\sum_{n \geq 1} \frac{1}{n} f\left(t^{n}\right),
$$

and in case of convergence, it holds that

$$
f(t) \leq \ln h(t) \leq g(t) .
$$

Proof. We have

$$
\ln h(t)=\sum_{n \geq 1} a_{n} \ln \frac{1}{1-t^{n}}=\sum_{n \geq 1} a_{n}\left(t^{n}+\frac{1}{2} t^{2 n}+\frac{1}{3} t^{3 n}+\ldots\right),
$$

showing the first equality. This also gives $f(t) \leq \ln h(t)$. In the case that $g(t)$ converges, it is given by

$$
g(t)=\sum_{n \geq 1} a_{n}\left(t^{n}+t^{2 n}+t^{3 n}+\ldots\right)
$$

and thus $\ln h(t) \leq g(t)$. 
Lemma 2. Suppose that the number $a_{m}$ of generators in dimension $m$ is bounded by a fixed polynomial in $m$. Then the radius of convergence of $h(t), f(t)$ and $g(t)$ is 1 .

Proof. We recall that an infinite product $\prod\left(1+u_{n}\right)$ is absolutely convergent if and only if $\sum u_{n}$ is. In our case, $u_{n}=t^{n} /\left(1-t^{n}\right)=t^{n}+t^{2 n}+t^{3 n}+\ldots$ and thus $h(t)$ is absolutely convergent if and only if $\sum b_{m} t^{m}$ is. Because of $b_{m} \leq a_{1}+a_{2}+\ldots+a_{m}$, $b_{m}$ is also bounded by a polynomial in $m$. Hence

$$
\limsup _{m} \sqrt[m]{b_{m}}=1
$$

(assuming that infinitely many of the $a_{n}$ are non-zero) and $\rho=1$.

Lemma 3. Suppose that there are $N>0$ and $n>0$ such that for all $k \geq 0$, there is some $i$ with $N+k n<i \leq N+(k+1) n$ and $a_{i}>0$ (i.e., above dimension $N$ there always exists at least one generator after $n$ steps). Then

$$
\lim _{s \rightarrow \infty} \ln h\left(1-e^{-s}\right) / s^{r}=\infty
$$

for all $r \geq 0$.

Proof. We have $\ln h(t) \geq f(t) \geq t^{N}\left(t^{n}+t^{2 n}+t^{3 n}+\ldots\right)=t^{N+n}\left(1-t^{n}\right)^{-1}$ and with $\left(1-t^{n}\right)=(1-t)\left(1+t+t^{2}+\ldots+t^{n-1}\right)$, we get

$$
\lim _{s \rightarrow \infty} \ln h\left(1-e^{-s}\right) / s^{r} \geq \frac{1}{n} \lim _{t \rightarrow 1^{-}}(1-t)^{-1} / s^{r}=\lim _{s \rightarrow \infty} e^{s} / s^{r}=\infty .
$$

Thus the gaps of dimensions without generators (i.e., $a_{i}=0$ ) of $H^{*}$ have to become larger and larger in order that $\lim _{s \rightarrow \infty} \ln h\left(1-e^{-s}\right) / s^{r}$ has a chance to be zero.

Corollary 4. Let $X$ be 1-connected of finite type and assume that there are $N>0$ and $n>0$ such that for all $k \geq 0$, there is some $x_{i} \in H^{i}(X ; \mathbb{Z} / p)$ with $N+k n<$ $i \leq N+(k+1) n$ such that the $x_{i}$ generate a polynomial subalgebra of $H^{*}(X ; \mathbb{Z} / p)$. Then $\pi_{*}(X) \otimes \mathbb{Z} / p$ is non-trivial in arbitrarily high dimensions.

Proof. By the last lemma, the growth rate of $X$ is infinite (or even $\rho(X)<1$ ).

This applies to stable classifying spaces $B U, B O, B S p, B S p i n$, etc. (where we know this result already by Bott periodicity, of course), but also to their iterated suspensions.

Our non-triviality theorem is an unstable result. In particular, one can ask if infinitely many of the non-trivial homotopy groups survive the process of stabilization.

In the stable case we can prove non-triviality by Serre's method only for the case of fast growth. We define "finite type", the $\bmod p$ Poincaré series and $\rho(X)$ of a spectrum $X$ as in the unstable case.

We recall the Poincaré series of the Steenrod algebra mod $p$ :

$$
h(H \mathbb{Z} / p, t)= \begin{cases}\prod_{n \geq 1}\left(1-t^{2^{n}-1}\right)^{-1} & \text { for } p=2, \\ \prod_{n \geq 1}\left(1-t^{2\left(p^{n}-1\right)}\right)^{-1} \prod_{i \geq 0}\left(1+t^{2 p^{i}-1}\right) & \text { for } p>2,\end{cases}
$$


which follows from the explicit structure of its dual algebra computed by J. Milnor. Furthermore, the admissible monomials in the Steenrod algebra that do not start with the Bockstein $\beta$ form a basis of $H^{*}(H \mathbb{Z} ; \mathbb{Z} / p)$, which yields

$$
h(H \mathbb{Z} / p, t)=(1+t) h(H \mathbb{Z} ; t) .
$$

In particular, $\rho(H \mathbb{Z} / p, t)=\rho(H \mathbb{Z}, t)=1$.

Theorem 3. Let $X$ be a connected spectrum of finite type. Assume that $\rho(X)<1$, or that $\rho(X)=1$ and that the function $h(X, t) / h(H \mathbb{Z}, t)$ is unbounded. Then the stable homotopy groups $\pi_{*}(X) \otimes \mathbb{Z} / p$ are non-trivial in arbitrarily high dimensions.

Proof. Let $Y$ be a connected spectrum of finite type with stable homotopy groups $\pi_{k}$ satisfying that $\pi_{k} \otimes \mathbb{Z} / p=0$ for $k>N$. The stable Postnikov tower of spectra $\Sigma^{k+1} H \pi_{k+1} \rightarrow Y^{(k+1)} \rightarrow Y^{(k)}$ gives

$$
h(Y, t) \leq t^{1} h\left(H \pi_{1}, t\right)+t^{2} h\left(H \pi_{2}, t\right)+\ldots+t^{N} h\left(H \pi_{N}, t\right)=p(t) h(H \mathbb{Z}, t),
$$

where $p(t)$ is a polynomial in $t$ of order at most $N+1$. The coefficients of $p(t)$ can be determined from the free ranks and $p$-torsion ranks of $\pi_{1}, \ldots, \pi_{N}$. Thus $\rho(Y) \geq 1$ and the function $h(Y, t) / h(H \mathbb{Z}, t)$ is bounded by the polynomial $p(t)$ on $0 \leq t \leq 1$.

We remark that it does not seem to be possible to obtain a lower bound for the Poincaré series of a stable finite Postnikov system by Serre's method. The lower bound obtained by considering the Whitehead tower of $Y$ has the form $h(Y, t) \geq$ $q(t) h(H \mathbb{Z}, t)$ with some polynomial $q(t)$, but $q(t)$ does not need to be positive on $0 \leq t \leq 1$.

Corollary 5. Let $X$ be a connected ring spectrum of finite type and assume that there are $N>0$ and $n>0$ such that for all $k \geq 0$, there is some $x_{i} \in H_{i}(X ; \mathbb{Z} / p)$ with $N+k n<i \leq N+(k+1) n$ such that the $x_{i}$ generate a polynomial subalgebra of $H_{*}(X ; \mathbb{Z} / p)$. Then the stable homotopy groups $\pi_{*}(X) \otimes \mathbb{Z} / p$ are non-trivial in arbitrarily high dimensions.

Proof. We show that $h(X, t) / h(H \mathbb{Z}, t)$ is unbounded. For simplicity, we show this only for $p=2$, but the proof also works for odd $p$ with slight modifications. We have

$$
\begin{aligned}
& \frac{h(X, t)}{h(H \mathbb{Z}, t)} \geq \frac{t^{N} \prod_{k \geq 1}\left(1-t^{k n}\right)^{-1}}{\prod_{k \geq 1}\left(1-t^{2^{k}-1}\right)^{-1}} \\
& \geq t^{N} \prod_{k \geq 1} \frac{1+t+t^{2}+\ldots+t^{2^{k}-2}}{1+t+t^{2}+\ldots+t^{k n-1}} .
\end{aligned}
$$

This diverges for $t \rightarrow 1^{-}$because the sum

$$
\sum_{k \geq 1}\left(\frac{2^{k}-1}{k n}-1\right)
$$

diverges.

\section{ACKNOWLEDGEMENT}

The author thanks the referee for valuable suggestions concerning further applications of the main theorem. 


\section{REFERENCES}

[B] D.J. Benson, Representations and Cohomology II: Cohomology of Groups and Modules, Cambridge studies in advanced mathematics, Vol 31 (1991) MR 93g:20099

[BK] A.K. Bousfield, D.M. Kan, Homotopy Limits, Completions and Localizations, Lect. Notes Math., Vol 304, Springer (1972) MR 51:1825

[L] Ran Levi, On Finite Groups and Homotopy Theory, Memoirs of the AMS, Vol 118, No 567 (1995) MR 96c:55019]

[MN] C.A. McGibbon, J.A. Neisendorfer, On the homotopy groups of a finite dimensional space, Comm. Math. Helvetici 59, 253-257 (1984) MR 86b:55015

[S] J.P. Serre, Cohomologie Modulo 2 des Complexes d'Eilenberg-MacLane, Comment. Math. Helv., Vol 27, 198-232 (1953) MR 15:643c

[U] Yoshio Umeda, A Remark on a Theorem of J.P. Serre, Proc. Japan Acad., Vol 35, 563-566 (1959) MR 22:12521

Mathematisches Forschungsinstitut Oberwolfach, Lorenzenhof, 77709 OBerwolfachWalke, Germany

E-mail address: klaus@mfo.de

$U R L$ : www.mfo.de/Staff/klaus.html 